\title{
Streptococcus pluranimalium sp. nov., from cattle and other animals
}

\author{
L. A. Devriese, ${ }^{1}$ P. Vandamme, ${ }^{2,3}$ M. D. Collins, ${ }^{4}$ N. Alvarez, ${ }^{4}$ B. Pot, ${ }^{2}$ \\ J. Hommez, ${ }^{5}$ P. Butaye ${ }^{1}$ and F. Haesebrouck ${ }^{1}$
}

Author for correspondence: L. A. Devriese. Tel: +3292647435 . Fax: +3292647494.
e-mail: luc.devriese@rug.ac.be

\footnotetext{
1 Faculty of Veterinary Medicine, University of Ghent, Salisburylaan 133, B9820 Merelbeke, Belgium

2 Faculty of Sciences, University of Ghent, Ghent, Belgium

3 University Hospital, University of Antwerp, Edegem, Belgium

4 Department of Food Science and Technology, University of Reading, Reading, UK

5 Regional Veterinary Investigation Centre, Torhout, Belgium
}

\begin{abstract}
Strains from subclinical mastitis, from the genital tract and tonsils of cattle, from tonsils of a goat and a cat and from the crop and the respiratory tract of canaries were found to constitute a new streptococcal species, for which the name Streptococcus pluranimalium sp. nov. is proposed. Sequencing of 165 rRNA showed that Streptococcus thoraltensis and Streptococcus hyovaginalis were its closest known phylogenetic relatives. The new species showed some phenotypic resemblance to the poorly described species Streptococcus acidominimus, but whole-cell protein analysis and 16S rRNA sequencing revealed that the new species was only distantly related to the type strain of $S$. acidominimus. Identification of these bacteria, which showed heterogeneous biochemical reaction patterns, was most reliably made by whole-cell protein analysis. Nevertheless, a number of biochemical reactions can be used to differentiate S. pluranimalium from other animal streptococci. Strain LMG 14177', isolated from mastitic milk of a dairy cow, was designated as the type strain of $S$. pluranimalium sp. nov.
\end{abstract}

Keywords: Streptococcus pluranimalium sp. nov., mastitis, genital tract flora, domestic animals

\section{INTRODUCTION}

During an investigation of unidentified aesculinhydrolysing mastitis streptococci, a number of aesculin-hydrolysing isolates were found to share many characteristics with certain strains from the genital tract and tonsils of cows, as well as with strains from very diverse animal hosts. Streptococci from bovine mastitis reacting positively in aesculin-containing isolation media are traditionally grouped with Streptococcus uberis in routine diagnostic bacteriology. Upon closer inspection, however, these bacteria are frequently found to be very different from this major streptococcal pathogen, especially when they originate from subclinical mastitis (Bentley et al., 1993, McDonald \& McDonald, 1976). In this paper, we report the results of a polyphasic study of a group of unknown aesculin-hydrolysing mastitis strains and of similar strains from other body sites as well as from

\footnotetext{
Abbreviations: AMC, 7-amido-4-methyl-coumarin; $M U$, methylumbelliferyl.

The GenBank accession number for the 165 rRNA sequence of Streptococcus
} pluranimalium sp. nov. strain LMG $14177^{\top}$ is $Y 18026$. other animal hosts. On the basis of the results of this study, a new species Streptococcus pluranimalium sp. nov. is described.

Many of the phenotypic characteristics of the strains investigated were found to correspond to those of the poorly described species Streptococcus acidominimus, which has been reported to occur in the bovine vagina and udder (Ayers \& Mudge, 1922; Hardie, 1986). Particular attention was given, therefore, to the differentiation of the new species from $S$. acidominimus, as well as from other phenotypically similar or phylogenetically related streptococcal species from animals.

\section{METHODS}

Strains. Twenty-three strains (Table 1) were investigated and compared with reference strains of known species. The strains had been isolated from the genital tract (cervix, vaginal mucus) and from subclinical mastitis in cows, from lungs and from the crop of canaries and from the tonsils of cattle, a cat and a goat. One strain (LMG 14257) was isolated from the cervix of a cow in Germany. LMG 14256, another bovine vaginal strain, originated from France. All others were from animals of different farms or owners in Belgium. 
Table 1. Strains of Streptococcus pluranimalium investigated in this study

Abbreviations used are LMG (BCCM/LMG Culture Collection, Laboratorium voor Microbiologie, Universiteit Gent, Belgium), CCUG (Culture Collection of Göteborg, Department of Clinical Microbiology, Göteborg, Sweden), L.A.D. (L. A. Devriese) and J.H. (J. Hommez).

\begin{tabular}{|llll|}
\hline \multicolumn{2}{|c}{ Strain number } & Depositor & \multicolumn{1}{c|}{ Isolation } \\
\cline { 1 - 2 } LMG & As received & & \\
\cline { 1 - 2 } 13589 & Ton444 & L.A.D. & Goat, tonsil \\
13592 & Ton200 & L.A.D. & Cat, tonsil \\
13612 & Ton427 & L.A.D. & Calf, tonsil \\
13613 & Ton263 & L.A.D. & Calf, tonsil \\
13614 & Ton213 & L.A.D. & Calf, tonsil \\
13615 & STR820 & J.H. & Cow, mastitis \\
13623 & Ton512 & L.A.D. & Calf, tonsil \\
13632 & Ton403 & L.A.D. & Calf, tonsil \\
13649 & K24 & L.A.D. & Canary, crop \\
14099 & D153 & L.A.D. & Cow, vagina \\
$14177^{\text {T }}$ & T70 & L.A.D. & Cow, mastitis \\
14256 & CCUG 11675 & CCUG & Cow, vagina \\
14257 & CCUG 28589 & CCUG & Cow, cervix \\
14385 & ESC101 & J.H. & Cow, mastitis \\
14482 & D54 & J.H. & Cow, vagina \\
14483 & D53 & J.H. & Cow, vagina \\
14827 & D244 & J.H. & Cow, vagina \\
14829 & D38 & J.H. & Cow, vagina \\
14831 & $94 / 241$ & L.A.D. & Canary, lung \\
15722 & POK3 & L.A.D. & Canary, pox lesion \\
16621 & T1368 & J.H. & Cow, mastitis \\
16622 & T1387 & J.H. & Cow, mastitis \\
16653 & M95/2121 & L.A.D. & Canary, lung \\
\cline { 1 - 2 } & & & \\
\hline
\end{tabular}

SDS-PAGE of whole-cell protein. Preparation of cellular protein extracts, PAGE, densitometric analysis, normalization of the protein profiles and numerical analysis were performed as described previously (Pot et al., 1994; Vandamme et al., 1998) by using the GeLCOMPAR 4.0 software package (Applied Maths). The similarity between all pairs of traces was expressed by the Pearson product moment correlation coefficient converted, for convenience, to a percentage similarity value.

Determination of the DNA $\mathbf{G}+\mathbf{C}$ content. The mean DNA $\mathrm{G}+\mathrm{C}$ content of strain $\mathrm{LMG} 14177^{\mathrm{T}}$ was determined as described by Mesbah et al. (1989). DNA was enzymically degraded into nucleosides and the nucleoside mixture obtained was then separated by HPLC using a Waters SymmetryShield C8 column thermostatted at $37^{\circ} \mathrm{C}$. The solvent was $0.02 \mathrm{M} \mathrm{NH}_{4} \mathrm{H}_{2} \mathrm{PO}_{4}$ (pH 4.0) with $1.5 \%$ acetonitrile. Non-methylated lambda phage DNA (Sigma) was used as the calibration reference.

16S rRNA gene sequence analysis. A large fragment of the 16S rRNA gene (corresponding to positions 30-1521 of the Escherichia coli $16 \mathrm{~S}$ rRNA gene) was amplified by PCR and sequenced directly by using a Dye-Deoxy terminator cycle sequencing kit (Applied Biosystems) and an automatic DNA sequencer (model 373A; Applied Biosystems). The sequences of the closest relatives of the unknown strains were retrieved from the GenBank or Ribosomal Database Project (RDP) databases and aligned with the newly determined sequences by using the program PILEUP (Devereux et al., 1984). The resulting multiple sequence alignment was corrected manually and a phylogenetic tree was constructed according to the neighbour-joining method with the program NEIGHBOR (Felsenstein, 1989). The stability of grouping was assessed by bootstrap analysis (200 replicates) (Felsenstein, 1989). In addition, parsimony analysis was performed (Felsenstein, 1989).

Growth characteristics and biochemical activity. These were studied as described previously (Devriese et al., 1997). Additionally, biochemical activity was tested in the BBL CRYSTAL Gram-positive kit (Becton Dickinson).

\section{RESULTS}

\section{SDS-PAGE of whole-cell protein}

A numerical analysis of the whole-cell protein patterns of all of the $S$. pluranimalium and $S$. acidominimus strains, together with reference strains of Streptococcus thoraltensis and Streptococcus hyovaginalis obtained from a previous study (Devriese et al., 1997), is shown in Fig. 1. Three clusters of strains are clearly distinguished. The $S$. acidominimus type strain, NCFB $2025^{\mathrm{T}}$, occupies a distinct position. One cluster consists of all $S$. pluranimalium strains, grouping above a

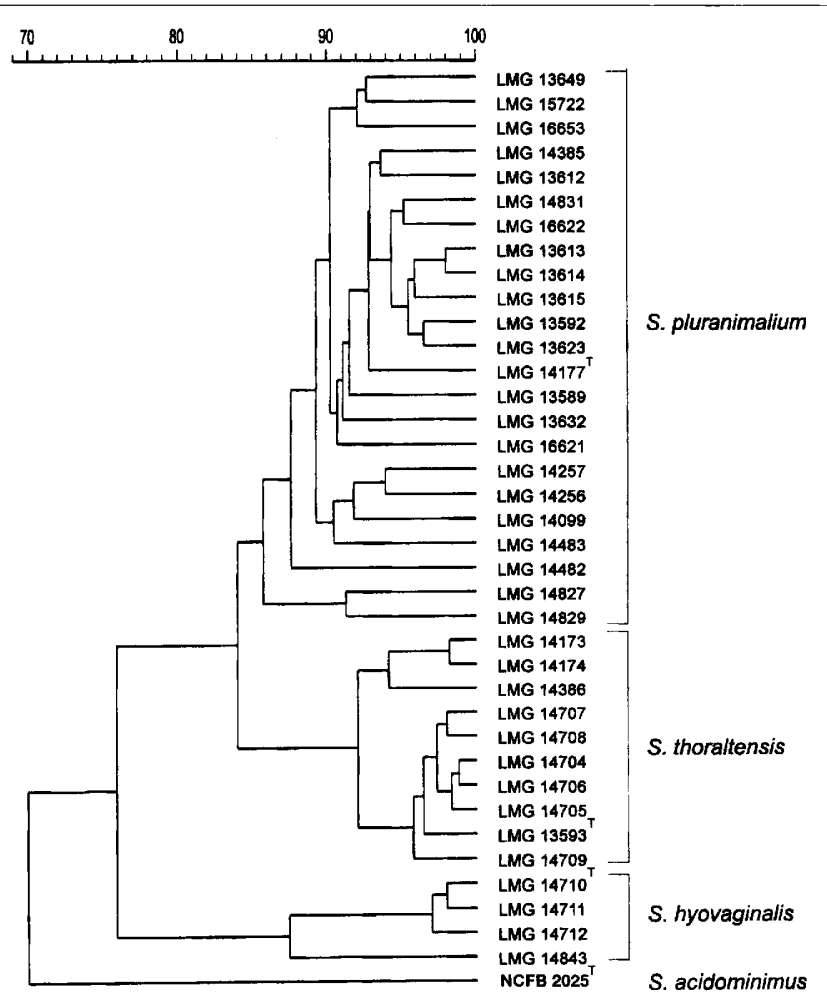

Fig. 1. Dendrogram derived from the unweighted pair group mean linkage of correlation coefficients (expressed as percentages) between whole-cell protein patterns of $S$. pluranimalium strains, the $S$. acidominimus type strain and strains belonging to the phylogenetically related species $S$. thoraltensis and S. hyovaginalis. 


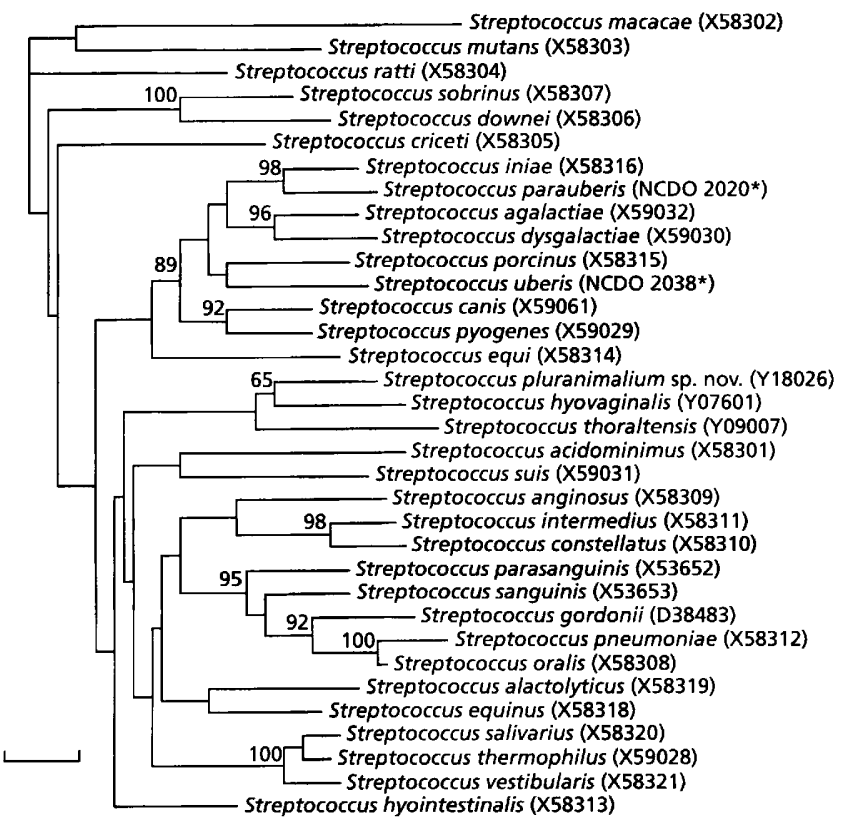

Fig. 2. Unrooted tree constructed by the neighbour-joining method based on 165 rRNA sequences showing the phylogenetic position of Streptococcus pluranimalium within the genus Streptococcus. *, Sequence from IFR database. Bar represents $1 \%$ sequence divergence. Only bootstrap values of $90 \%$ or more are shown, except for the value of $65 \%$ for the species $S$. pluranimalium and $S$. hyovaginalis.

correlation level of $85 \%$. The remaining clusters are formed by the $S$. thoraltensis and $S$. hyovaginalis strains, grouping above correlation levels of 91 and $87 \%$, respectively.

\section{Determination of the DNA G + C content}

The mean DNA G + C content of strain LMG $14177^{\mathrm{T}}$ was $38.5 \mathrm{~mol} \%$.

\section{5 rRNA gene sequence analysis}

The 16S rRNA genes of two strains (LMG 14257 and LMG $14177^{\mathrm{T}}$ ) were amplified by PCR and sequenced directly. Almost complete sequences ( $>1400$ bases) were determined for each strain and pairwise analysis revealed $99.9 \%$ sequence identity (corresponding to one nucleotide difference). Sequence searches of the GenBank and RDP databases showed that the unknown organisms were most closely related to $S$. hyovaginalis (3\% sequence divergence) and $S$. thoraltensis $(3.5 \%$ divergence). A tree, constructed by using the neighbour-joining method, depicting the position of the bacterium (as exemplified by $\mathrm{LMG}$ $14177^{\mathrm{T}}$ ) within the genus Streptococcus is shown in Fig. 2. The treeing analysis confirmed $S$. hyovaginalis as the closest relative of LMG $14177^{\mathrm{T}}$, followed by $S$. thoraltensis. All other streptococcal species were distantly related. Parsimony analysis confirmed the results of the neighbour-joining analysis.
Table 2. Variable characteristics of Streptococcus pluranimalium strains

\begin{tabular}{|c|c|}
\hline Characteristic & $\begin{array}{c}\text { No. positive/ } \\
\text { no. tested }\end{array}$ \\
\hline Growth in $6.5 \% \mathrm{NaCl}$ broth $(2 \mathrm{~d})$ & $11 / 23$ \\
\hline Aesculin hydrolysis on Edward's agar & $21 / 23$ \\
\hline Brown discoloration on Edward's agar & $16 / 23$ \\
\hline Aesculin hydrolysis in API 20 STREP & $12 / 23$ \\
\hline \multicolumn{2}{|l|}{ Activity of: } \\
\hline Pyrrolidonyl arylamidase & $7 / 23$ \\
\hline$\alpha$-Galactosidase & $10 / 23$ \\
\hline$\beta$-Galactosidase & $3 / 23$ \\
\hline$\beta$-Glucuronidase & $9 / 23$ \\
\hline$\beta$-Glucosidase & $15 / 23$ \\
\hline Alkaline phosphatase & $20 / 23$ \\
\hline Arginine dihydrolase & $4 / 23$ \\
\hline$N$-Acetyl $\beta$-glucosaminidase & $6 / 23$ \\
\hline \multicolumn{2}{|l|}{ Hydrolysis of: } \\
\hline 4-MU $\beta$-glucoside & $7 / 13$ \\
\hline L-Valine AMC & $11 / 13$ \\
\hline 4-MU $\alpha$-D-glucoside & $12 / 13$ \\
\hline L-Pyroglutamic acid AMC & $3 / 13$ \\
\hline L-Arginine AMC & $12 / 13$ \\
\hline 4-MU $N$-acetyl- $\beta$-D-glucosaminide & $7 / 13$ \\
\hline 4-MU $\beta$-D-glucuronide & $8 / 13$ \\
\hline L-Isoleucine AMC & $11 / 13$ \\
\hline$p$-Nitrophenyl $\beta$-D-glucoside & $8 / 13$ \\
\hline$p$-Nitrophenyl $\beta$-D-cellobioside & $8 / 13$ \\
\hline Proline and leucine $p$-nitroanilide & $11 / 13$ \\
\hline \multicolumn{2}{|l|}{ Production of acid from: } \\
\hline$N$-Acetylglucosamine & $17 / 23$ \\
\hline Amygdalin & $10 / 23$ \\
\hline Arbutin & $11 / 23$ \\
\hline Cellobiose & $13 / 23$ \\
\hline Galactose & $15 / 23$ \\
\hline$\beta$-Gentiobiose & $12 / 23$ \\
\hline Lactose & $15 / 23$ \\
\hline D-Mannose & $17 / 23$ \\
\hline Mannitol & $12 / 23$ \\
\hline Maltose & $18 / 23$ \\
\hline Melezitose & $10 / 23$ \\
\hline Melibiose & $6 / 23$ \\
\hline Methyl $\beta$-glucopyranoside & $13 / 23$ \\
\hline Raffinose & $11 / 23$ \\
\hline Ribose & $11 / 23$ \\
\hline Saccharose & $18 / 23$ \\
\hline Salicin & $13 / 23$ \\
\hline Sorbitol & $9 / 23$ \\
\hline D-Tagatose & $4 / 23$ \\
\hline
\end{tabular}

AMC, 7-amido-4-methyl-coumarin; MU, methylumbelliferyl.

\section{Growth characteristics and resistance}

All strains were Gram-positive cocci and formed chains, pairs or small groups. The chain lengths were very variable. The cells were relatively small, definitely smaller than those of the well-known $\beta$-haemolytic 
Table 3. Characteristics that differ between bovine strains of Streptococcus pluranimalium from the genital tract and from other sources

No. positive/no. tested is shown for each characteristic.

\begin{tabular}{|lcr|}
\hline Characteristic & $\begin{array}{c}\text { Genital tract } \\
\text { strains }\end{array}$ & $\begin{array}{c}\text { Other } \\
\text { strains }\end{array}$ \\
\hline Growth in 6.5\% NaCl broth & $0 / 7$ & $8 / 10$ \\
Browning on Edward's medium & $2 / 7$ & $9 / 10$ \\
Activity of: & & \\
$\quad \beta$-Glucuronidase & $6 / 7$ & $1 / 10$ \\
$\beta$-Glucosidase & $1 / 7$ & $8 / 10$ \\
Production of acid from: & & \\
Amygdalin & $0 / 7$ & $10 / 10$ \\
Arbutin & $0 / 7$ & $10 / 10$ \\
Cellobiose & $0 / 7$ & $10 / 10$ \\
$\beta$-Gentiobiose & $0 / 7$ & $10 / 10$ \\
Mannitol & $1 / 7$ & $9 / 10$ \\
Melezitose & $0 / 7$ & $8 / 10$ \\
Raffinose & $0 / 7$ & $10 / 10$ \\
Ribose & $0 / 7$ & $8 / 10$ \\
Sorbitol & $0 / 7$ & $8 / 10$ \\
\hline
\end{tabular}

pyogenic cocci. Most strains produced precipitates with clear supernatants in Brain Heart Infusion broth after $1 \mathrm{~d}$ incubation. Growth at $42^{\circ} \mathrm{C}$ was similar to that at $37^{\circ} \mathrm{C}$. The strains did not grow or grew only faintly after $1 \mathrm{~d}$ at $25^{\circ} \mathrm{C}$. Slightly enhanced growth was noted with certain strains when plates were incubated in $5 \% \mathrm{CO}_{2}$ at $37^{\circ} \mathrm{C}$. On Columbia sheep blood agar, colonies were not pigmented and were regular, transparent and small, less than $1 \mathrm{~mm}$ in diameter. All strains were $\alpha$-haemolytic. Colonies on blood agar were usually surrounded by very pronounced greening zones with sharply demarcated edges. The strains were not motile.

Growth in $6.5 \% \mathrm{NaCl}$ broth was variable (Table 2). All strains grew on Edward's medium and all but two lysed aesculin on this medium. All but five bovine genital tract strains and two strains from other sources produced colonies with strong browning or blackening effects on the medium surrounding the bacterial growth. They grew to very small colonies on kanamycin aesculin azide agar but did not produce blackening in this medium. Growth on Slanetz and Bartley medium was poor or absent.

The $S$. acidominimus type strain NCFB $2025^{\mathrm{T}}$ showed similar growth and resistance characteristics. It was moderately capnophilic and did not grow on blood agar at $42^{\circ} \mathrm{C}$.

\section{Biochemical activity}

All strains reacted positively in the following tests: hippurate hydrolysis, activity of leucine arylamidase and alanine-phenylalanine-proline arylamidase, hydrolysis of 4-methylumbelliferyl (MU)-phosphate, Lphenylalanine 7-amido-4-methyl-coumarin (AMC) and L-tryptophan AMC, acid production from glucose, fructose and trehalose. Carbohydrate reactions were very weak and slow to develop in certain strains. All were negative in the amylase plate test, in VogesProskauer tests, in tests for catalase and $\beta$-mannosidase activity, hydrolysis of $p$-nitrophenyl $\alpha$-Dmaltoside, urease activity and acid production from

Table 4. Characteristics differentiating Streptococcus pluranimalium from phylogenetically related and phenotypically similar species

$\mathrm{v}$, Variable reaction: $\mathrm{v}+$, most strains $(>75 \%)$ positive; $\mathrm{v}-$, most strains $(>75 \%)$ negative. $\mathrm{D}$, Reaction depends on strain origin (see Table 3).

\begin{tabular}{|c|c|c|c|c|c|}
\hline Characteristic & S. pluranimalium & S. thoraltensis & S. hyovaginalis & S. suis & S. acidominimus \\
\hline Strong greening on blood agar & + & - & - & - & + \\
\hline Voges-Proskauer reaction & - & + & + & - & - \\
\hline Hippurate hydrolysis & + & $\mathrm{v}$ & + & $v-$ & - \\
\hline \multicolumn{6}{|l|}{ Activity of: } \\
\hline$\beta$-Glucuronidase & D & + & - & $v+$ & + \\
\hline Alkaline phosphatase & $v+$ & $\mathrm{v}$ & + & $v-$ & - \\
\hline \multicolumn{6}{|l|}{ Production of acid from: } \\
\hline L-Arabinose & - & + & - & - & - \\
\hline Glycogen & - & - & - & + & - \\
\hline Inulin & - & + & - & $\mathrm{v}+$ & Delayed + \\
\hline Mannitol & D & + & - & $v-$ & Delayed + \\
\hline Ribose & $\mathrm{D}$ & + & $\mathrm{v}$ & - & - \\
\hline Trehalose & + & + & + & + & - \\
\hline Sorbitol & $\mathrm{D}$ & + & + & - & - \\
\hline Starch & - & + & - & + & - \\
\hline
\end{tabular}


adonitol, starch, D- and L-arabitol, D- and L-arabinose, D-cyclodextrin, 2- and 5-ketogluconate, dulcitol, erythritol, D- and L-fucose, methyl $\alpha$-D-glucoside, gluconate, glycogen, inositol, inulin, D-lyxose, methyl $\alpha$ D-mannoside, pullulan, rhamnose, L-sorbose, Dturanose, D- and L-xylose, xylitol and methyl $\beta$ xyloside. Variable reactions are listed in Table 2 . Among the bovine strains, a number of characteristics differed according to body location. The strains from vagina and cervix were biochemically less reactive than strains from subclinical mastitis and from tonsils. Only the $\beta$-glucuronidase reaction showed a clearly opposite tendency. These differing reactions are tabulated here (Table 3) because they facilitate biochemical identification.

Characteristics of the $S$. acidominimus type strain NCFB $2025^{\mathrm{T}}$ differing from the strains included in the present study are listed in Table 4 . In addition, it was noted that this strain did not react in tests for acid production from salicin and cellobiose. This is in contradiction to results described in the literature (Kilpper-Bälz et al., 1985).

\section{DISCUSSION}

In contrast to most streptococcal species, which are associated with only one or a few phylogenetically related animal hosts (Devriese, 1991), the strains investigated were found in very different animal host species. Moreover, the body locations of the species were unusually diverse: genital tract, udder and tonsils of mammals; crop, skin and respiratory tract of birds. SDS-PAGE analysis of whole-cell protein revealed that the strains were phenotypically related at the species level. The results described above, obtained by very different types of tests, all demonstrated that the strains included in this study belong to a new species of the genus Streptococcus. 16S rRNA gene sequence analysis demonstrated that strains LMG 14257 and LMG $14177^{\mathrm{T}}$ were closely related to each other genealogically and constituted a new subline within this genus. The bacteria described here displayed a specific association (Fig. 2) with the recently described species $S$. hyovaginalis and $S$. thoraltensis from sows. However, sequence divergence values of approximately $3 \%$ and $3.5 \%$, respectively, indicate that the isolates represent a hitherto unknown species. Although a precise correlation between 16S rRNA sequences and DNA-DNA pairing is not possible, the $3 \%$ or greater levels of $16 \mathrm{~S}$ rRNA sequence divergence between the unknown species and $S$. hyovaginalis and $S$. thoraltensis are supportive of a new species (Stackebrandt \& Goebel, 1994). The phylogenetic distinctiveness of the unknown bacterium is consistent with its separateness as revealed by whole-cell protein analysis and it is proposed that organisms of this species be designated Streptococcus pluranimalium sp. nov.

A special feature of the strains described here is the relatively large number of variable biochemical char- acteristics (Table 2). This metabolic variability possibly relates to the very diverse habitats of this species. This infra-specific aspect falls outside the scope of the present paper but clearly merits further study. The divergent reactions of the bovine genital (vagina and cervix) strains, as shown in Table 3 , are merely an indication of the possible existence within $S$. pluranimalium of ecovars showing typical host-specific or even site-specific characteristics, as is well known in other streptococci (Devriese, 1991). Although the species was associated with subclinical mastitis in dairy cows and was isolated from lesions in canaries, its pathogenic significance remains unclear. Moreover, the preponderance of bovine strains in the collection examined in this study is probably a reflection of the animal material at our disposal.

The presence of $S$. pluranimalium on blood-agar isolation plates can be suspected when colonies are surrounded by broad, sharply demarcated zones of strong greening haemolysis. On aesculin-containing blood agars such as Edward's medium, colonies are usually intensely brown or black, as is the surrounding medium. Characteristics useful in the differentiation of $S$. pluranimalium from phylogenetically related or phenotypically similar species are shown in Table 4. The usefulness of some of these characteristics may depend on the origin of the strains (Tables 3 and 4 ). $S$. pluranimalium may also be confused with strains of the Streptococcus bovis species group and even with the greening enterococci of the Streptococcus avium species group (Devriese et al., 1993) and with the lactococci. The negative Voges-Proskauer reaction of $S$. pluranimalium differentiates the new species unequivocally from these groups.

Certain strains (LMG 14256, = CCUG 11675; LMG 14257 , = CCUG 28589) were originally identified as $S$. acidominimus. It has long been recognized that the taxonomy of $S$. acidominimus is unsatisfactory and that the species as defined (Ayers \& Mudge, 1922; Hardie, 1986) embraces many misidentified strains. In view of these difficulties, the type strain $S$. acidominimus, NCFB $2025^{\mathrm{T}}$, which has been shown by $16 \mathrm{~S}$ rRNA sequence analysis to represent a separate species (Bentley et al., 1993), was included in our study. This strain differs markedly from $S$. pluranimalium (Figs 1 and 2, Table 4). Its biochemical characteristics, as determined here, were in agreement with the original species description. It is pertinent to note that they differed from those listed by Kilpper-Bälz et al. (1985) in not less than five of eleven tests.

\section{Description of Streptococcus pluranimalium sp. nov.}

Streptococcus pluranimalium (plur.an.im.al'i.um. L. adj. pluris many; L. n. gen. pl. animalium from animals; M.L. gen. n. pluranimalium from many animals).

Cells are small, Gram-positive cocci arranged in chains or groups. Non-motile. Grows at 37 and $42{ }^{\circ} \mathrm{C}$. Most strains produce precipitates and clear supernatants in 
broth. Colony size less than $1 \mathrm{~mm}$. Produces greening haemolysis. May grow in $6.5 \% \mathrm{NaCl}$ broth. Reacts positively in tests for hydrolysis of hippurate, 4-MUphosphate, L-phenylalanine AMC and L-tryptophan AMC, leucine arylamidase, alanine-phenylalanineproline arylamidase, 4-MU-phosphate hydrolysis, acid production from glucose, fructose and trehalose. Other carbohydrate reactions are very variable and, in some strains, weak and slow to develop. Negative in the Voges-Proskauer test. The DNA G $+\mathrm{C}$ content of the type strain is $38.5 \mathrm{~mol} \%$. The type strain is LMG $14177^{\mathrm{T}}$; this strain was isolated from subclinical mastitis in a cow.

\section{ACKNOWLEDGEMENTS}

We thank Arlette Van de Kerckhove, Johan Goris and Dirk Dewettinck for excellent assistance with different aspects of the study. P.V. is indebted to the Fund for Scientific Research - Vlaanderen (Belgium) for a position as a postdoctoral research fellow. We thank all depositors of strains listed in Table 1.

\section{REFERENCES}

Ayers, S. H. \& Mudge, C. S. (1922). The streptococci of the bovine udder. $J$ Infect Dis 31, 40-50.

Bentley, R. B., Leigh, J. A. \& Collins, M. D. (1993). Development and use of species-specific oligonucleotide probes for differentiation of Streptococcus uberis and Streptococcus parauberis. $J$ Clin Microbiol 31, 57-60.

Devereux, J., Haeberli, P. \& Smithies, O. (1984). A comprehensive set of sequence analysis programs for the VAX. Nucleic Acids Res 12, 387-395.

Devriese, L. A. (1991). Streptococcal ecovars associated with different animal species: epidemiological significance of serogroups and biotypes. $J$ Appl Microbiol 71, 478-483.
Devriese, L. A., Pot, B. \& Collins, M. D. (1993). Phenotypic identification of the genus Enterococcus and differentiation of phylogenetically distinct enterococcal species and species groups. J App/ Bacteriol 75, 399-408.

Devriese, L. A., Pot, B., Vandamme, P., Kersters, K., Collins, M. D., Alvarez, N., Haesebrouck, F. \& Hommez, J. (1997). Streptococcus hyovaginalis sp. nov. and Streptococcus thoraltensis sp. nov., from the genital tract of sows. Int J Syst Bacteriol 47, 1073-1077.

Felsenstein, J. (1989). PHYLIP - phylogeny inference package (version 3.2). Cladistics 5, 164-166.

Hardie, J. M. (1986). Other streptococci. In Bergey's Manual of Systematic Bacteriology, vol. 2, pp. 1068-1070. Edited by P. H. Sneath, N. S. Mair, M. S. Sharpe \& J. H. Holt. Baltimore: Williams \& Wilkins.

Kilpper-Bälz, R., Wenzig, P. \& Schleifer, K. H. (1985). Molecular relationships and classification of some viridans streptococci as Streptococcus oralis and emended description of Streptococcus oralis (Bridge and Sneath 1982). Int J Syst Bacteriol 35, 482-488.

McDonald, T. J. \& McDonald, J. S. (1976). Streptococci isolated from bovine intramammary infections. Am $J$ Vet Res 37, 377-381.

Mesbah, M., Premachandran, U. \& Whitman, W. B. (1989). Precise measurement of the $\mathrm{G}+\mathrm{C}$ content of deoxyribonucleic acid by high-performance liquid chromatography. Int $J$ Syst Bacteriol 39, 159-167.

Pot, B., Vandamme, P. \& Kersters, K. (1994). Analysis of electrophoretic whole-cell organism protein fingerprints. In Modern Microbial Methods. Chemical Methods in Bacterial Systematics, pp. 493-521. Edited by M. Goodfellow \& A. G. O'Donnell. Chichester: Wiley.

Stackebrandt, E. \& Goebel, B. M. (1994). Taxonomic note: a place for DNA-DNA reassociation and 16S rRNA sequence analysis in the present species definition in bacteriology. Int $J$ Syst Bacteriol 44, 846-849.

Vandamme, P., Torck, U., Falsen, E., Pot, B., Goossens, H. \& Kersters, K. (1998). Whole-cell protein electrophoretic analysis of viridans streptococci: evidence for heterogeneity among Streptococcus mitis biovars. Int J Syst Bacteriol 48, 117-125. 